\title{
Determinación de la influencia del contenido de humedad en el coeficiente de rozamiento interno de microesferas de cristal mediante ensayos de corte directo
}

\author{
F. Gallego-Gómez ${ }^{1}$, Manuel Moya ${ }^{2}$ \\ 1 Instituto de Ciencias de Materiales de Madrid (ICMM, CSIC). C/ Sor Juana Inés de la Cruz, 3, 28049 \\ Cantoblanco (Madrid); francisco.gallego@icmm.csic.es \\ 2 Universidad de Extremadura. Centro Universitario de Plasencia. Avda. Virgen del Puerto no 2, 10600 \\ Plasencia (Cáceres); manuelmi@unex.es
}

\begin{abstract}
Resumen: En este trabajo se realizaron ensayos de corte directo para determinar las curvas tensión-deformación de microesferas de cristal en un amplio intervalo de humedades. De esta forma se pudo determinar la variación del ángulo de rozamiento interno del material con la humedad. Además, se estudió la variación de la densidad del material, expresada como fracción sólida, con la carga normal aplicada y la humedad. En general, se determinó que, para cada una de las cargas normales aplicadas, que variaban entre 3,4 y $350 \mathrm{kPa}$, la fracción sólida oscilaba entre 0,47 y 0,74 , aproximadamente, aumentando progresivamente hacia valores asintóticos a medida que la carga normal aumentaba. Por otro lado, se observó que la fracción sólida alcanzaba valores muy altos en condiciones secas (0,69-0,74), disminuía sensiblemente $(0,48-0,68)$ al comenzar a añadirle agua en un intervalo bajo de humedad $(0,1-$ $5 \%)$, y aumentaba claramente a humedades elevadas, entre el 17 y el $20 \%(0,59-0,69$ a $\mathrm{H}=20 \%)$. Por su parte, de los ensayos de corte directo llevados a cabo se pudo comprobar que para bajos contenidos de humedad el material dilataba, mientras que éste se compactaba a altas humedades. Finalmente, se obtuvieron dos ángulos de rozamiento diferentes dependiendo de la región de carga normal aplicada, uno para cargas normales elevadas, entre 100 y $250 \mathrm{kPa}$, el otro para cargas normales bajas comprendidas entre 0 y $80 \mathrm{kPa}$.
\end{abstract}

Palabras clave: material granular, propiedades mecánicas, densidad, capilaridad, fracción sólida

\section{Introducción}

Hace mucho tiempo que se tiene constancia de la relación que existe entre el contenido de humedad y las propiedades mecánicas de materiales granulares. En condiciones de humedad las moléculas de agua se insertan entre los granos a través de las fuerzas de capilaridad, afectando de esta manera a la cohesión y al rozamiento del material [1-3]. De hecho, la cantidad de líquido presente en el medio granular determina el número y la forma de los puentes de capilaridad que se establecen entre los granos y, por lo tanto, los estados pendular, funicular y de capilaridad que se puedan dar. En ese sentido, a medida que un determinado material pasa de un estado húmedo a otro seco, o viceversa, se crean o se destruyen uniones capilares/puentes capilares/puentes líquidos entre partículas, determinando el comportamiento cohesivo del material, aspecto que se está investigando en los últimos años [4-7]. Además, es especialmente interesante comprender bien el comportamiento de material en las transiciones entre los 


\section{CONGRESO IBÉRICO DE AGROINGENIERÍA \\ X CONGRESSO IBÉRICO DE AGROENGENHARIA \\ 3 - 6 septiembre 2019, Huesca - España}

regímenes de humedad existentes, lo que ocurre habitualmente cuando se añaden muy pequeñas cantidades de agua que permiten alcanzar el régimen pendular, que determina el paso del estado seco al húmedo, o como ocurre en el paso del régimen funicular al de capilaridad cuando se dan condiciones de humedad próximas a las del estado de saturación. La aparición de las fuerzas de capilaridad entre partículas provoca resistencia a la tracción que afecta significativamente a la densidad del medio granular húmedo y su compactación cuando se somete a una carga externa. Estos aspectos determinan la resistencia a tracción, el rozamiento entre partículas, el papel lubricante del agua, la fracción sólida en el medio, etc., rigiendo el comportamiento de materiales granulares sometidos a tensiones normales y tangenciales, lo cual posee gran interés para una gran cantidad de campos naturales, científicos e industriales, desde la geología a la tecnología de materiales pulverulentos.

A pesar de lo expuesto en el párrafo anterior, habitualmente la investigación de las características mecánicas de materiales granulares y pulverulentos se limita al empleo de muestras secas [8-10] y, más raramente, pues hay relativamente muy pocos estudios al respecto, se centran en el estudio de la humedad empleando principalmente cantidades intermedias de líquido (habitualmente en el estado pendular, rara vez hasta el estado funicular) [11-13], olvidándose de lo que ocurre en las transiciones a contenidos de humedad muy bajos o muy altos, que es lo que ocurre en condiciones reales, como es el caso de las muestras de material granular no consolidadas. Por lo tanto, en la actualidad existe una carencia en la comprensión del comportamiento mecánico de materiales granulares húmedos no consolidados especialmente en contenidos de humedad críticos. Por este motivo, en este trabajo se va a realizar un estudio para determinar la influencia de la humedad en la resistencia al corte de microesferas de cristal de tamaño en torno a $100 \mu \mathrm{m}$, así como para conocer cómo influye ésta en la variación de la densidad de dicho material con la carga normal aplicada. La información que se obtenga puede ser de gran utilidad para diversas aplicaciones prácticas, desde el manejo de un determinado material granular a la evaluación del riesgo de materiales geológicos o habitualmente utilizados en la ingeniería civil.

\section{Materiales y métodos}

Como se ha comentado en el párrafo anterior, para la realización del presente trabajo se utilizaron microesferas de cristal de tamaño medio aproximado de $100 \mu \mathrm{m}$. Este material se utiliza frecuentemente como aditivo en multitud de aplicaciones, tales como adhesivos, masillas, revestimientos, resina epoxy, etc., permitiendo que el producto final que se obtiene pueda mejorar sus condiciones (protección frente a caídas, aislamiento térmico y auditivo, rigidez, reducción de la densidad, etc.)

\subsection{Preparación de la muestra}

Para la realización de los ensayos, en primer lugar se procedió a lavar repetidamente el material utilizado con agua destilada para eliminar las impurezas que pudiera contener éste, procediendo al secado de éste en estufa a $120^{\circ} \mathrm{C}$ durante 6 días, cribándolo posteriormente en un tamiz con tamaño de abertura de $220 \mu \mathrm{m}$ para eliminar los posibles agregados que pudieran haberse formado. Para certificar la ausencia de impurezas en la muestra, así como la suavidad de la superficie de las microesferas y la esfericidad de la mayoría de ellas, se escaneó todo el material en un microscopio electrónico (SEM). De esta manera, una vez que se añadió el líquido, pudieron descartarse los mecanismos cohesivos distintos de las fuerzas de capilaridad, como las fuerzas físico-químicas o la cementación química. Las superficies de los cristales son duras e insolubles en líquidos humectantes, como el agua, y a la vez el tamaño medio de las microesferas es suficientemente grande como para despreciar las fuerzas de Van der Waals o las 


\section{CONGRESO IBÉRICO DE AGROINGENIERÍA \\ X CONGRESSO IBÉRICO DE AGROENGENHARIA \\ 3 - 6 septiembre 2019, Huesca - España}

fuerzas electrostáticas, pero suficientemente pequeñas como para despreciar la fuerza gravitatoria en presencia de los puentes de capilaridad.

Para humedecer la muestra se utilizó agua destilada en laboratorio. El contenido de humedad se expresa como el porcentaje del peso de agua añadida con respecto al peso total (agua y microesferas). La cantidad de muestra elegida fue la misma en todos los ensayos llevados a cabo ( $250 \mathrm{~g})$, de modo que en un recipiente cerámico se depositaba ésta y se añadía la cantidad de agua necesaria para los distintos puntos de humedad que se deseaban ensayar, mezclando bien mediante movimientos circulares y durante un tiempo suficiente, alrededor de 10 minutos, para homogeneizar la muestra resultante utilizando para ello una varilla de cristal. En el proceso de homogeneización de la muestra se evitó en todo momento presionar a la muestra contra las paredes o el fondo del recipiente, o realizar movimientos excesivamente rápidos para que no se compactara ésta. El intervalo de humedad obtenido finalmente osciló entre el $0,1 \%$ y el $22 \%$. No se pudieron ensayar humedades superiores al $22 \%$ porque se producía una fase de separación del material, lo que denotaba que se alcanzaba el estado de saturación del material granular.

Durante la realización de los distintos ensayos se minimizó en todo momento la exposición de la muestra a la humedad y temperatura ambientes (la humedad relativa del aire fue inferior en todos los casos al 30\%). Además, tras completar cada tanda de ensayos con una misma muestra, ésta era depositada en el interior de una estufa para proceder a su secado a una temperatura de $105-110^{\circ} \mathrm{C}$ durante 24 horas.

\subsection{Densidad del material}

La densidad de la muestra fue determinada para todos los intervalos de humedad previstos, incluyendo el caso seco ( $0 \%$ de humedad). Para ello se utilizó la fracción sólida, definida como la relación entre el volumen de las microesferas de cristal y el volumen total de la muestra en condiciones de humedad, tal como se muestra en la expresión 1 :

Siendo

$$
f=\frac{V_{\text {microesferas }}\left(\mathrm{cm}^{3}\right)}{V_{\text {Total }}\left(\mathrm{cm}^{3}\right)}=\frac{\frac{\text { Peso seco }(g)}{\rho_{s}\left(\frac{g}{\mathrm{~cm}^{3}}\right)}}{V_{\text {Total }}\left(\mathrm{cm}^{3}\right)}
$$

$\mathrm{f}$ = fracción sólida, expresada en tanto por uno

$\rho$ s la densidad de las microesferas totalmente secas. Su valor, aplicando el método del picnómetro, es de $2,55 \mathrm{~g} / \mathrm{cm}^{3}$.

$\mathrm{V}_{\text {microesferas }}=$ volumen de la muestra de microesferas secas utilizadas en cada ensayo de corte directo, expresado en $\mathrm{cm}^{3}$.

$V_{\text {total }}=$ volumen total ocupado por la muestra de microesferas para cada estado de humedad ensayado, expresado en $\mathrm{cm}^{3}$.

Se trata de un parámetro indicativo de la compactación de la muestra antes de la realización de los ensayos de corte directo. Para su determinación, la muestra, a la que se le había añadido previamente la cantidad de agua necesaria para alcanzar la humedad prevista en cada caso, se colocó en el interior de una caja de corte circular de $10 \mathrm{~cm}$ de diámetro, procediendo a medir la altura inicial alcanzada por ella en el interior de la caja de corte. A partir de ese momento la muestra fue sometida a diferentes presiones normales que variaban en un intervalo entre 0 y 350 $\mathrm{kPa}$, incrementando la carga aplicada una vez que la muestra se consolidada a la carga aplicada en ese momento. El descenso provocado en la altura de la muestra situada en el interior de la caja de corte como consecuencia de la compactación sufrida por ésta al aplicarle los diferentes escalones de carga normal fue determinado mediante un sensor de desplazamiento, de modo que con la aplicación informática utilizada para tal efecto se conocía perfectamente dicha altura del material y, por lo tanto, el volumen ocupado por la muestra húmeda en cada escalón. 


\subsection{Ensayo de corte directo}

En el laboratorio de geotecnia del Centro Universitario de Plasencia se dispone de un aparato de corte directo semiautomático con el que se realizaron los ensayos previstos en el presente trabajo y que es el que se muestra en la figura 1:

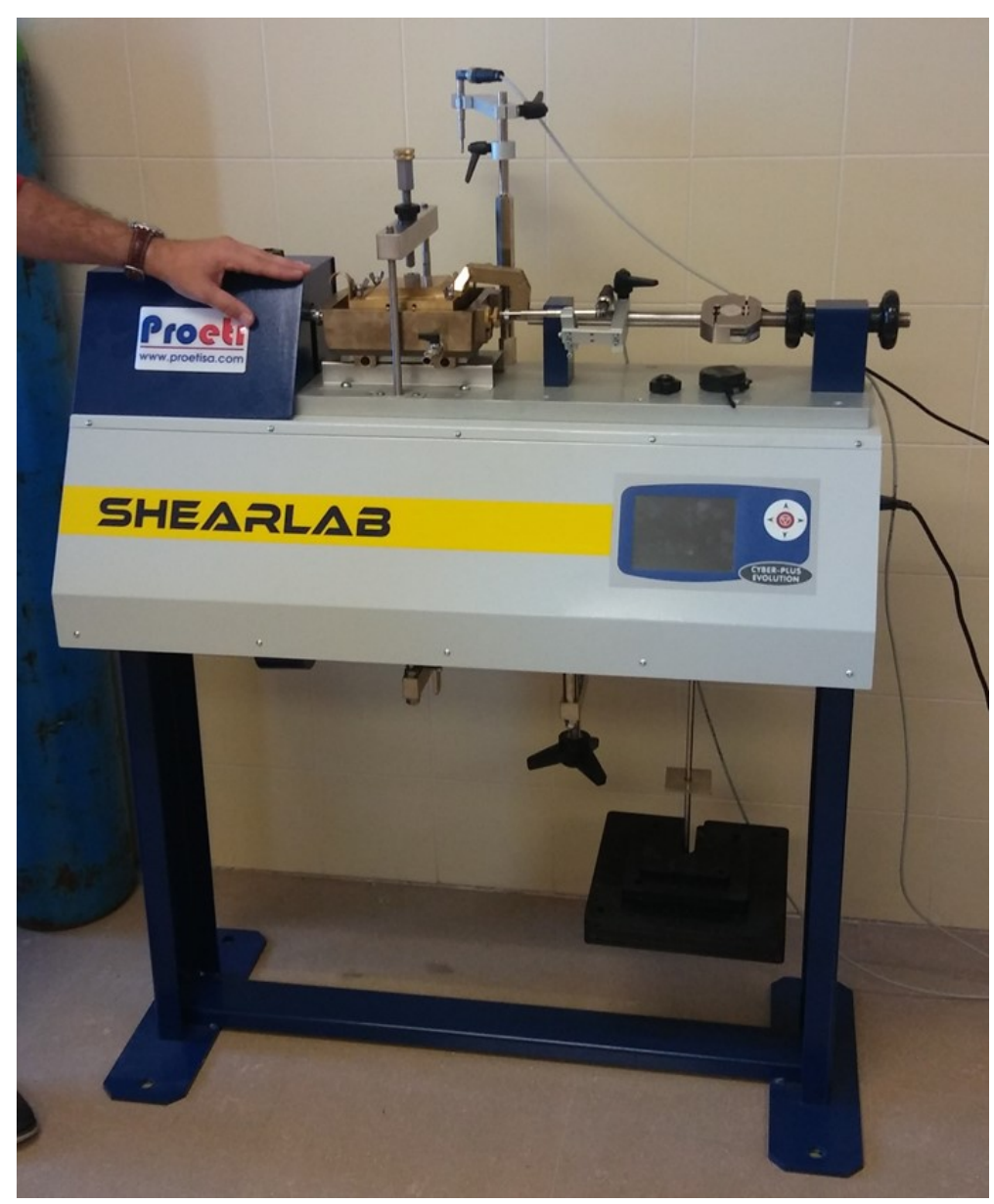

Figura 1. Aparato de corte directo utilizado en el laboratorio de geotecnia del Centro Universitario de Plasencia

En el presente trabajo se utilizó la caja de corte circular de $10 \mathrm{~cm}$ de diámetro y $3 \mathrm{~cm}$ de altura, cumpliéndose así el requisito de que la relación entre el tamaño de la caja y el tamaño medio de las partículas de la muestra utilizada sea superior a 40 para evitar el efecto escala. Para el desarrollo del ensayo, en primer lugar se procedió al llenado de la caja de corte con la muestra de microesferas humedecidas previamente al punto de humedad que se deseaba ensayar en cada momento, evitando el uso de técnicas de vibrado o de tapeo para no compactar la muestra. Una vez distribuida ésta uniformemente por toda la superficie de la caja de corte se colocaban sobre ella una piedra porosa, una rejilla, además de la propia tapa, piezas todas ellas del mismo diámetro, aplicando así una carga vertical inicial de 3,43 kPa (que era la primera carga vertical considerada). Sobre la tapa se colocaba un sensor de desplazamiento vertical y, a su vez, una célula de carga proporcionaba la fuerza tangencial que se iba aplicando en cada momento, permitiendo obtener así la relación entre la deformación horizontal y la tensión tangencial, además de conocer la deformación vertical de la muestra. La velocidad de ensayo fue 0,63 $\mathrm{mm} / \mathrm{min}$ y el desplazamiento máximo permitido de $12 \mathrm{~mm}$, ambos suficientes para que se produjera la rotura del material en el menor tiempo posible, evitando así que se pudiera secar la 


\section{CONGRESO IBÉRICO DE AGROINGENIERÍA \\ X CONGRESSO IBÉRICO DE AGROENGENHARIA \\ 3 - 6 septiembre 2019, Huesca - España}

muestra en la serie completa realizada a las distintas cargas normales ensayadas. Con esta primera carga se procedió a realizar el ensayo de corte directo, de modo que una vez finalizado éste se repetía el proceso vaciando con sumo cuidado la caja de corte para no perder material, volviendo a colocarlo en su interior de forma similar a la descrita anteriormente, colocando dicha caja en el carro del aparato de corte directo, poniendo la piedra porosa, la rejilla y la tapa y colocando para la siguiente y sucesivas cargas crecientes el bastidor que posee un brazo multiplicador de carga (se seleccionó la posición de multiplicar por 10 dicha carga) y un juego de pesas para alcanzar la presión vertical deseada en cada caso. En total se establecieron entre 8 y 10 escalones de carga en función del contenido de humedad de la muestra, alcanzando en todo caso una presión normal máxima de 350 $\mathrm{kPa}$, como se comentó anteriormente. El ensayo se repitió dos veces para cada una de las cargas verticales consideradas con el fin de obtener un valor medio fiable.

Para cada uno de los puntos de humedad seleccionados, una vez realizada la serie completa de cargas verticales prevista para ellos, con los datos proporcionados por la célula de carga y el transductor de desplazamiento vertical, además del conocimiento que se tenía sobre la velocidad de corte seleccionada (deformación horizontal), se pudo representar la envolvente de MohrCoulomb, determinando así el ángulo de rozamiento interno del material y la cohesión aparente. Además, pudieron representarse las curvas tensión-deformación horizontal y las de deformación vertical-deformación horizontal, como se verá en la siguiente sección dedicada a la exposición de resultados su posterior discusión.

\section{Resultados y discusión}

\subsection{Fracción sólida}

En primer lugar se van a mostrar los resultados obtenidos para la fracción sólida de las microesferas de cristal. En la figura 2 se observa la tendencia general seguida cuando se representa la fracción sólida en el eje de ordenadas, expresada en tanto por uno, y la presión normal (en $\mathrm{kPa}$ ), en el eje de abscisas. En este caso concreto la humedad aplicada a la muestra era del $10 \%$. Resultados bastante similares se han obtenido para esta misma muestra con contenidos de humedad diferentes, variando éste entre el 0,5\% y el 22\%.

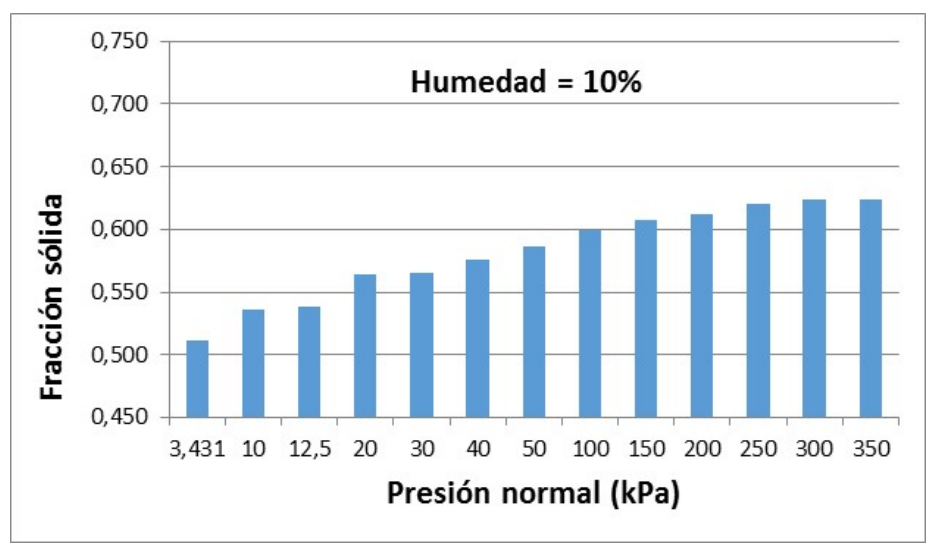

Figura 2. Variación de la fracción sólida con la presión normal aplicada para muestra humedecida al $10 \%$

Como se puede observar en la gráfica anterior, la fracción sólida va creciendo más o menos uniformemente a presiones bajas y su tendencia se va suavizando hasta tender a un valor asintótico a presiones elevadas. Este comportamiento es lógico teniendo en cuenta que a medida que se aplican cargas mayores la muestra se va compactando pero lo hace un poquito menos cada vez porque el volumen de aire que hay dentro de la muestra va siendo menor a medida que aumenta la presión normal aplicada, como se corrobora en [14]. 
Por su parte, la figura 3 muestra la variación de la fracción sólida con el contenido de agua, en los puntos de humedad seleccionados para tal fin, y para cada una de las presiones normales aplicadas a la muestra.

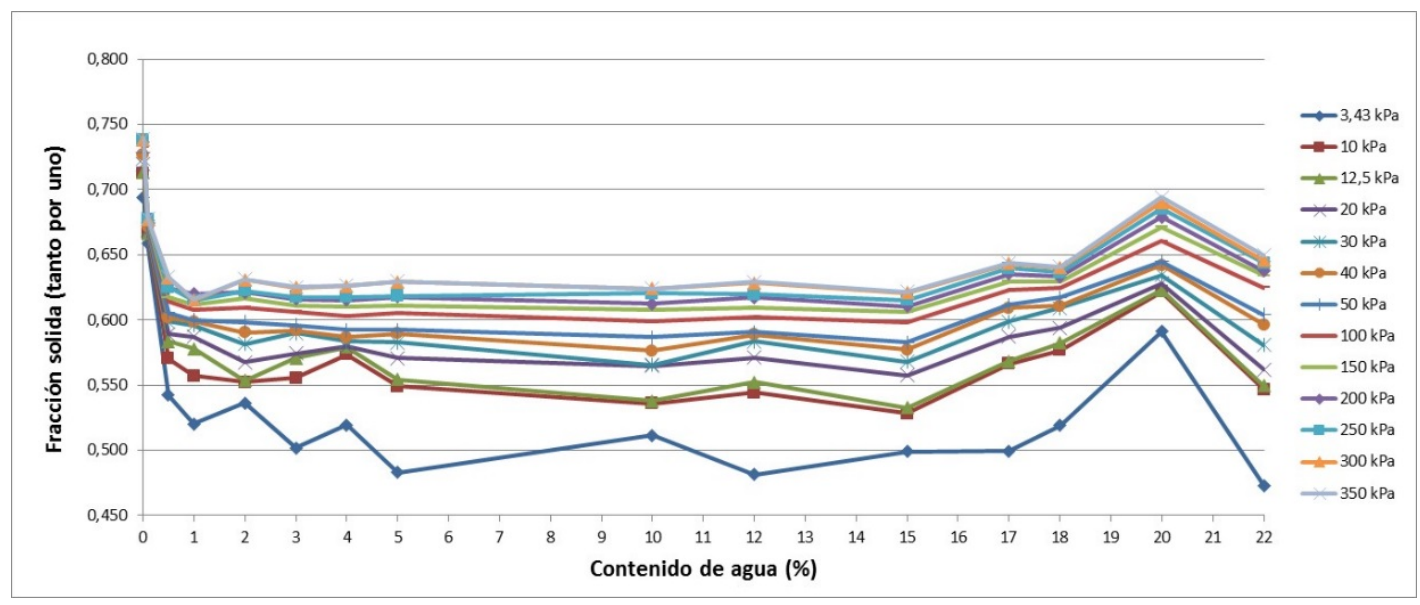

Figura 3. Variación de la fracción sólida con la humedad para cada una de las presiones normales aplicadas a la muestra

De entrada, en esta figura se corrobora que la fracción sólida varía claramente en función de la humedad que posee la muestra en cada momento. Como se puede observar, si partimos de los valores obtenidos para este parámetro con la muestra totalmente seca ( $0 \%$ de humedad), que oscilan entre 0,69 y 0,74 , dichos valores disminuyen sensiblemente con contenidos de humedad muy bajos (en el intervalo comprendido entre el 0,1 y el 5\%, aproximadamente, según casos), oscilando éstos entre 0,48 y 0,68. Para humedades más elevadas, entre el 5 y el $15 \%$ de agua, aproximadamente, la fracción sólida se estabiliza experimentando leves subidas o bajadas en su valor en función de la carga normal aplicada, incrementándose su valor a partir del 15\% y alcanzando un valor "pico" al $20 \%$ de humedad (valor que en ningún caso supera al alcanzado con la muestra totalmente seca), oscilando en este punto entre 0,59 y 0,69, valor que va disminuyendo seguidamente de manera sensible al $22 \%$ de humedad, situándose entre 0,47 y 0,65.

De manera semicuantitativa la dependencia de la fracción sólida con respecto a la humedad podría explicarse considerando el número y la distribución de los puentes de capilaridad que se crean por la adición de agua. En condiciones totalmente secas, $\mathrm{f}$ alcanza un valor de 0,69 para una tensión normal de 3,43 $\mathrm{kPa}$, llegando a un valor de 0,74 a $350 \mathrm{kPa}$. Sin embargo, estos valores pasan a ser de 0,66 y 0,68 para una tensiones normales de 3,43 y $350 \mathrm{kPa}$, respectivamente, con una cantidad mínima de agua $(0,1 \%$, a temperatura ambiente). Esto es lógico pues en condiciones secas no existen fuerzas cohesivas significativas entre las esferas presentes en la muestra, estando ésta relativamente suelta de manera aleatoria, mientras que con la aplicación de una pequeña carga normal $(3,43 \mathrm{kPa})$ se logra una la ligera compactación del material, comenzando a crearse arcos estables entre las diferentes partículas, incrementando la cantidad de poros grandes [15]. El claro descenso de f, por debajo de 0,48 al 5\% de humedad, sugiere que la baja viscosidad y la gran tensión superficial del agua le asignen un papel fundamental a las fuerzas de capilaridad atractivas ( $\mathrm{y}$, por lo tanto, a la aparición de estructuras granulares sueltas sostenidas por el rozamiento estático entre partículas) sobre los efectos de la lubricación en los puntos de contacto entre partículas.

Por el contrario, a humedades más altas se forman puentes de líquido entre las esferas y, por lo tanto, las cada vez mayores tensiones tangenciales que se producen a continuación en el material llevan a una compactación progresiva y global de éste. Esta tendencia se produciría hasta llegar al 20\% de humedad, pues a humedades superiores se alcanza la saturación del 


\section{CONGRESO IBÉRICO DE AGROINGENIERÍA \\ X CONGRESSO IBÉRICO DE AGROENGENHARIA \\ 3 - 6 septiembre 2019, Huesca - España}

material. Este hecho puede atribuirse al comienzo de coalescencia de los puentes líquidos y la ocupación por el líquido de los huecos entre partículas, lo que lleva a una incipiente de la succión capilar (o presión de Laplace) dentro del material, dando paso al denominado régimen funicular. Finalmente, al continuar con la adición de agua, los huecos que quedan entre las esferas son ocupados por ésta y la cohesión del material se induce sólo por la succión capilar en la superficie externa, alcanzando el estado capilar. En consecuencia, la resistencia a tracción del material disminuye posteriormente y así lo hace también la compactación, hasta $\mathrm{f}=0,47$. Se ha de tener en cuenta que, al compararlo con el estado seco, la presencia de agua en abundancia entre las esferas induce una separación adicional de las partículas sólidas, disminuyendo la fracción sólida a pesar de la succión capilar (que es nula en el material granular seco).

\subsection{Ensayo de corte directo}

Las curvas tensión-deformación obtenidas a partir de los ensayos de corte directo llevados a cabo muestran una tendencia clara en función del contenido de agua y la tensión normal aplicada, y lo mismo ocurre con las curvas de deformación obtenidas (deformación vertical frente a desplazamiento horizontal), tal como se aprecia en la figura 4.

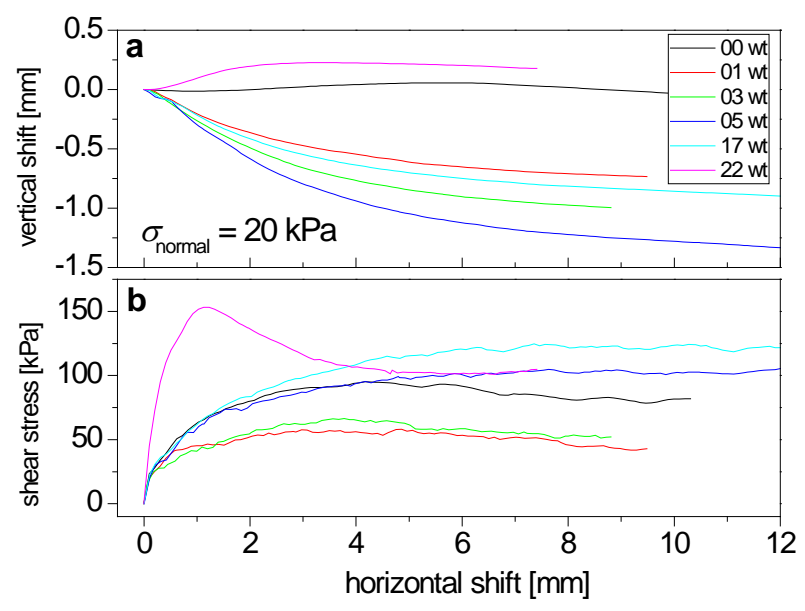

(a)

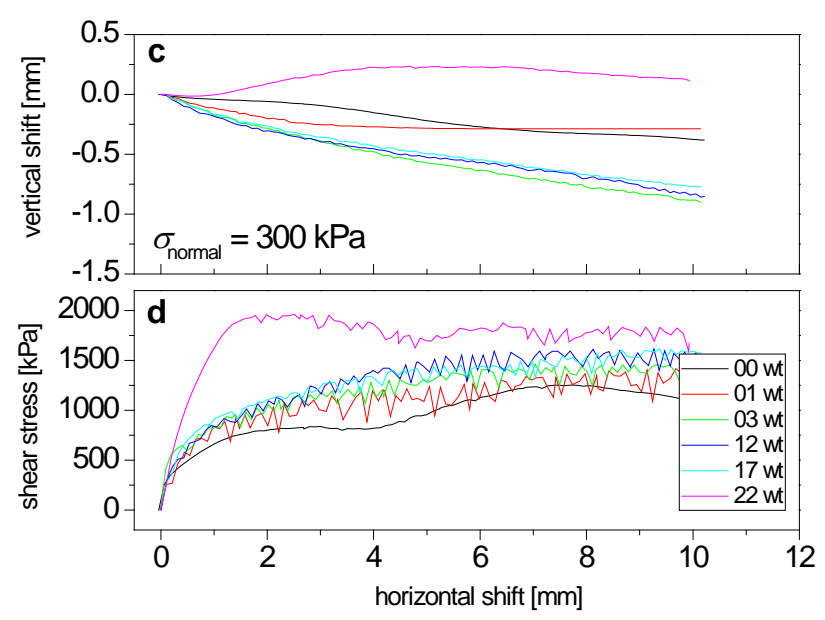

(b)

Figura 4. Curvas tensión-deformación (gráfica inferior) y curvas de deformación (gráfica superior) en función de la humedad y de la tensión vertical aplicadas obtenidas en los ensayos de corte directo a) Tensión normal de $20 \mathrm{kPa}$. b) Tensión normal de $300 \mathrm{kPa}$. 


\section{CONGRESO IBÉRICO DE AGROINGENIERÍA \\ X CONGRESSO IBÉRICO DE AGROENGENHARIA \\ 3 - 6 septiembre 2019, Huesca - España}

En la figura anterior, las dos gráficas de deformación parecen mostrar la dependencia de la deformación experimentada por la muestra con la densidad de ésta, parámetro que fue tratado en el apartado anterior. Así, mientras las muestras en seco mostraban una cierta dilatación $(d>0)$, algo típico en los sistemas granulares densos [8,9], con las muestras húmedas el comportamiento es el opuesto, es decir, se va comprimiendo la muestra $(\mathrm{d}<0)$ a medida que se va produciendo el corte ya que la baja fracción sólida dejaba espacios vacíos para que se pudieran acomodar las partículas que estaban en movimiento. Sin embargo, para grandes humedades el grado de compactación disminuye hasta que, al final, la muestra dilata claramente en el estado de capilaridad ( $\mathrm{H}=22 \%)$, lo que puede atribuirse a la gran cantidad de agua que ocupa los huecos vacíos que hay entre las partículas sólidas. Estas tendencias se acentúan a bajas tensiones normales, mientras que con tensiones normales mayores se induce una mayor densidad de la muestra, reduciéndose por lo tanto el grado de compactación experimentado durante el corte.

Por su parte, las curvas tensión-deformación mostraron de manera general un pequeño pero progresivo incremento de la resistencia al corte a medida que se iba produciendo éste tendiendo a un valor máximo asintótico, casi constante. En general, la resistencia al corte aumentaba con la humedad, especialmente en el caso de bajas tensiones normales, en el que la dependencia de la fracción sólida con respecto a la humedad parece ser clave (figura 4a), mientras que esta influencia era menor a mayores tensiones normales (figura $4 \mathrm{~b}$ ). Por otro lado, con muestra seca $(\mathrm{H}=0 \%)$ la tensión tangencial alcanzada era intermedia a bajas tensiones normales pero fue la menor a tensiones normales elevadas. Este comportamiento puede justificarse si se tiene en cuenta tanto la configuración de la muestra seca en comparación con la de las muestras húmedas como la ausencia de la resistencia a tracción en el caso seco. Por un lado, a bajas tensiones normales la muestra seca densa puede mostrar mayor resistencia al corte que la ofrecida por las muestras húmedas sueltas hasta que la resistencia a tracción de las últimas sea suficientemente alta al aumentar la humedad. Por otro lado, a mayores tensiones normales la densidad de las muestras, tanto la de la seca como las de las húmedas, es similar, lo que unido a la ausencia de resistencia a tracción facilita el corte de las muestras secas. Por su parte, la muestra saturada $(\mathrm{H}=22 \%)$ mostró una curva de corte claramente diferente a las anteriores pues en ella la tensión tangencial alcanzó rápidamente un valor pico a desplazamientos horizontales bajos (> $2 \mathrm{~mm}$ ), disminuyendo inmediatamente después y estabilizándose relativamente a desplazamientos horizontales más altos (tendiendo a un valor asintótico inferior en cualquier caso al máximo alcanzado anteriormente). A su vez, al analizar lo que ocurre con la curva de deformación vertical en condiciones de saturación se puede comprobar que el comportamiento de la muestra difiere sensiblemente de la tendencia que se observa con muestras a humedades inferiores ya que en este caso la muestra dilata en todo momento, incrementándose el grado de dilatación a bajos desplazamientos horizontales y suavizándose esta tendencia a medida que éstos aumentan.

La evolución de las tensiones tangenciales de las muestras ensayadas a medida que avanzaban los ensayos se produjo de manera bastante suave a bajas tensiones normales, hasta los $100 \mathrm{kPa}$, aproximadamente, pero a tensiones normales superiores las curvas obtenidas se tornaron más irregulares, experimentando los valores de la tensión tangencial rápidos ascensos y descensos a pequeños incrementos del desplazamiento horizontal (lo que en la terminología anglosajona se denomina fenómeno "slip-stick"), fenómeno que ocurre con bastante asiduidad durante el corte de medios granulares cuando se aplican cargas elevadas [16-18]. La única excepción a este comportamiento se produjo con la muestra totalmente seca $(\mathrm{H}=0 \%)$, incluso cuando para ésta se aplicaban las tensiones normales máximas (350 kPa). En el caso de muestra saturada $(\mathrm{H}=22 \%)$ también se observó este fenómeno si bien en este caso las caídas en los valores de la tensión tangencial no eran tan bruscas. 


\section{CONGRESO IBÉRICO DE AGROINGENIERÍA \\ X CONGRESSO IBÉRICO DE AGROENGENHARIA \\ 3 - 6 septiembre 2019, Huesca - España}

La figura 5 muestra las envolventes de Mohr-Coulomb obtenidas a partir de la media de los valores máximos alcanzados para la tensión tangencial en los distintos ensayos de corte directo realizados a diferentes tensiones normales y a distintas humedades. La pendiente de las diferentes envolventes proporciona el valor del ángulo de rozamiento interno para cada caso, mientras que el punto en el que éstas cortan al eje de ordenadas determina el valor de la cohesión aparente del material.
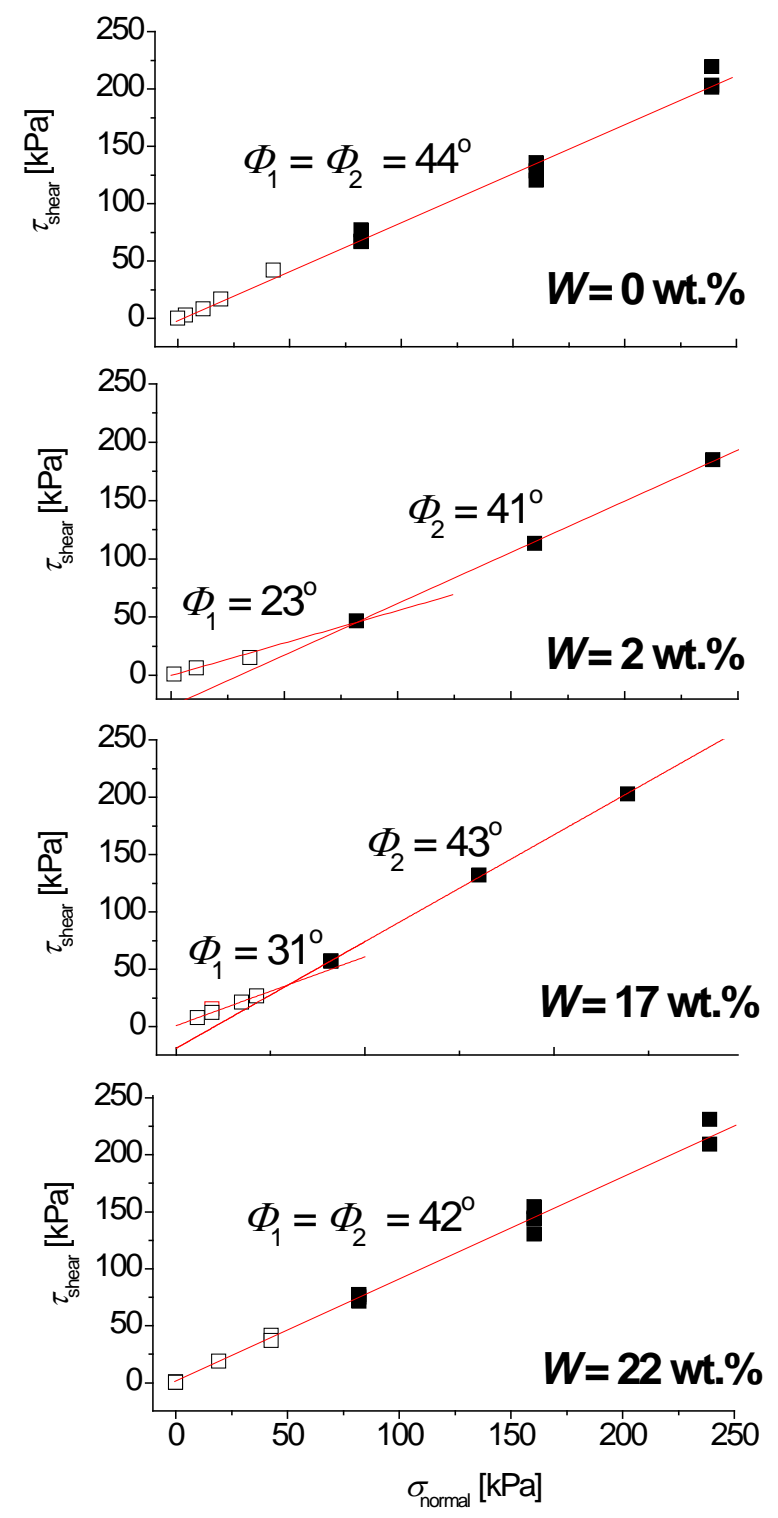

Figura 5. Envolventes de Mohr obtenidas a distintas humedades.

De la figura 5 se deduce que tanto para la muestra seca $(\mathrm{H}=0 \%)$ como para la muestra saturada $(\mathrm{H}=22 \%)$ se obtiene una única recta, proporcionando valores del ángulo de rozamiento interno de $44^{\circ}$ y $42^{\circ}$, respectivamente. Sin embargo, para valores intermedios de humedad se manifiestan dos tendencias diferenciadas, una a bajas tensiones normales (entre 3,43 y $80 \mathrm{kPa}$ ) y otra a altas tensiones normales (entre 100 y $250 \mathrm{kPa}$ ), obteniéndose por lo tanto dos valores diferentes para el ángulo de rozamiento interno en estas condiciones, a los que denominaremos $\phi_{1}$ y $\phi_{2}$, respectivamente, oscilando éste entre 20 y $45^{\circ}$ en los distintos intervalos de humedad ensayados. Los valores más altos del ángulo de rozamiento interno aquí obtenidos 


\section{CONGRESO IBÉRICO DE AGROINGENIERÍA \\ X CONGRESSO IBÉRICO DE AGROENGENHARIA \\ 3- 6 septiembre 2019, Huesca - España}

son en general superiores a los obtenidos con materiales agrícolas granulares y pulverulentos [8-10].

La figura 6 muestra la variación del ángulo de rozamiento interno con el contenido de humedad. La imagen de la izquierda es para bajas tensiones normales, donde se obtiene $\phi_{1}$, mientras que la figura de la derecha corresponde a altas tensiones normales, donde se obtiene $\phi 2$.

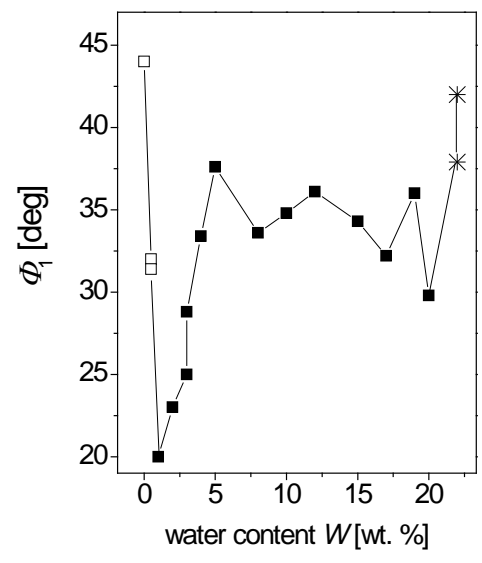

(a)

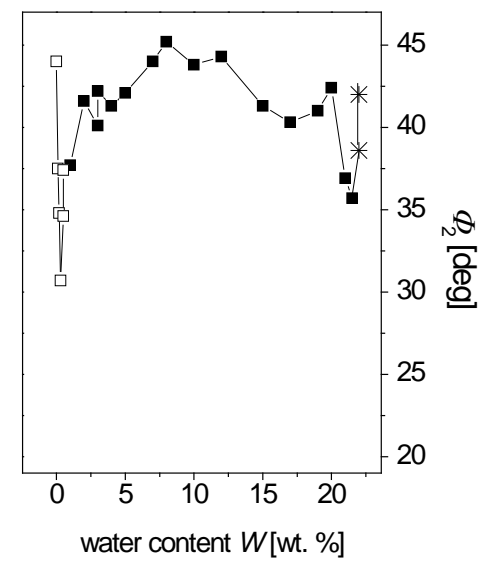

(b)

Figura 6. Variación del ángulo de rozamiento interno con la humedad. a) Para bajas tensiones normales $\left(\phi_{1}\right)$. b) Para altas tensiones normales $\left(\phi_{2}\right)$.

De la figura anterior se deduce que a bajas humedades la pendiente de la envolvente de Mohr-Coulomb tendía a suavizarse, especialmente notable para una humedad del 1\%, para la que el ángulo de rozamiento interno obtenido fue de $20^{\circ}$, menos de la mitad de los $44^{\circ}$ obtenidos para la muestra seca $(\mathrm{H}=0 \%)$. Este descenso se debe a que con una pequeña adición de agua se favorece el corte de la muestra, disminuyendo la resistencia ofrecida por ésta. Por esta misma razón, a bajas tensiones normales los valores obtenidos para $\phi$ en materiales granulares no consolidados siempre fueron menores con muestras humedecidas moderadamente, si bien en estos casos el valor del ángulo de rozamiento interno fue mayor que el obtenido al $1 \%$ de humedad.

Por su parte, al analizar lo que ocurre con el ángulo de rozamiento interno a tensiones normales elevadas $\left(\Phi_{2}\right)$, se constata igualmente su dependencia de la humedad pero con algunas diferencias significativas con respecto a lo observado a bajas tensiones normales. Así, el valor mínimo es mayor que el obtenido en el caso anterior $\left(30^{\circ}\right.$ a $\left.\mathrm{H}=0,5 \%\right)$ y a humedades intermedias los valores obtenidos eran más parecidos a los máximos alcanzados en los extremos $\mathrm{y}$, por lo tanto, generalmente superiores a los obtenidos en materiales agrícolas granulares y pulverulentos [8-10]. Además, a elevadas tensiones normales la humectación del material granular no consolidado no conlleva necesariamente una reducción de la resistencia al corte, como ocurría a bajas cargas normales.

\section{Conclusiones}

La determinación de las propiedades mecánicas de materiales granulares en distintas condiciones de humedad es esencial en ámbitos tan diversos como la geología, la ingeniería civil, la industria farmacéutica o las industrias agrarias, entre otros. De estas propiedades dependerán tanto la seguridad de las instalaciones correspondientes en las que se almacenen, como los costes de las operaciones de manejo, pues éstos pueden variar sensiblemente en 


\section{CONGRESO IBÉRICO DE AGROINGENIERÍA \\ X CONGRESSO IBÉRICO DE AGROENGENHARIA \\ 3 - 6 septiembre 2019, Huesca - España}

función de la eficiencia de los procesos correspondientes. A pesar de esto, son muy pocos los estudios científicos realizados hasta el momento sobre esta cuestión, por lo que se hace preciso investigar más a fondo.

A tenor de los ensayos llevados a cabo en este trabajo se puede concluir que la fracción sólida depende de la humedad a la que se encuentre sometida la muestra correspondiente, hallándose intervalos de humedad en los que este parámetro oscila sensiblemente. En general, se observa que el valor obtenido para este parámetro es máximo con muestras totalmente secas, alcanzando valores entre 0,69 y 0,74. Entre el 0,1 y el 5\% de humedad la fracción sólida cae sensiblemente con respecto a los valores obtenidos en condiciones totalmente secas $(0,48-0,68)$, permaneciendo más o menos estable a humedades intermedias (5-15\%), para aumentar al $20 \%$ de humedad $(0,59-0,69)$ y disminuir finalmente en condiciones de saturación $(0,47-0,65$ al $22 \%$ de humedad).

De los ensayos de corte directo realizados cabe destacar, en primer lugar, la relación de dependencia que se observa entre el ángulo de rozamiento interno y la humedad de la muestra. En las envolventes de Mohr-Coulomb obtenidas en las muestras secas y saturadas (0 y $22 \%$ de humedad, respectivamente) la tendencia seguida fue la de una única recta, obteniéndose ángulos de rozamiento interno de $44^{\circ}$ y $42^{\circ}$, respectivamente. Sin embargo, a humedades intermedias $(0,1-20 \%)$ se observaron dos tendencias diferentes en la curva, una a bajas tensiones normales $(0-80 \mathrm{kPa})$ y otra a elevadas tensiones normales $(100-250 \mathrm{kPa})$, dando lugar a dos ángulos de rozamiento (๑1 y $\odot 2$, respectivamente). A cargas normales bajas, para una humedad del $1 \%$ se obtuvo un valor mínimo $\odot 1=20^{\circ}$, aumentando progresivamente los valores de $\odot$ con la humedad en intervalos intermedios de esta última (2-20\%), mientras que para una humedad del $0,5 \%$ se obtuvo un valor mínimo $\odot 2=30^{\circ}$, encontrándose los valores correspondientes de $\odot$ a humedades intermedias en valores parecidos a los obtenidos al 0 y $22 \%$ de humedad.

\section{Referencias}

1. Davies R.M., El-Okene A.M. Moisture-dependent physical properties of soybeans. International Agrophysics. 2009, 23, 299-303.

2. Frye K.M., Marone C. Effect of humidity on granular friction at room temperature. Journal of Geophysical Research. 2002, 107(11), 13 pp.

3. Nokhodchi A. An overview of the effect of moisture on compaction and compression. Pharmaceutical Technology. 2005, 29(1), 46-66.

4. Delenne J.Y., El Youssoufi M.S., Cherblanc F., Bénet J.C. Mechanical behavior and failure of cohesive granular materials. International Journal for Numerical and Analytical Methods in Geomechanics. 2004, 28, 1577-1594.

5. Delenne J.Y., Soulié F., El Youssoufi M.S., Radjai F. From liquid to solid bonding in cohesive granular media. Mechanics of Materials. 2011, 43, 529-537.

6. Topin V., Delenne J.Y., Radjai F., Brendel L., Mabille F. Strength and fracture of cemented granular matter. The European Physical Journal E. 2007, 23, 413-429.

7. Jiang M., Yu H., Harris D. Bond rolling resistance and its effect on yielding of bonded granulates by DEM analyses. International Journal for Numerical and Analytical Methods in Geomechanics. 2006, 30(8), 723-761.

8. Moya M., Ayuga F., Guaita M., Aguado, P.J. Mechanical properties of granular agricultural materials. Transations of the ASAE. 2002, 45(5), 1569-1577.

9. Moya M., Guaita M., Aguado P.J., Ayuga, F. Mechanical properties of granular agricultural materials. Part 2. Transactions of the ASABE. 2006, 49(2), 479-489.

10. Ramírez A., Moya M., Ayuga F. Determination of the mechanical properties of powdered agricultural products and sugar. Particle and Particle Systems Characterization. 2009, 26(4), 220-230.

11. Louati H., Oulahna D., de Ryck A. Apparent friction and cohesion of a partially wet granular material in steady-state shear. Powder Technology. 2015, 278, 65-71. 


\section{CONGRESO IBÉRICO DE AGROINGENIERÍA \\ X CONGRESSO IBÉRICO DE AGROENGENHARIA \\ 3 - 6 septiembre 2019, Huesca - España}

12. Landi G., Barletta D., Poletto M. Modelling and experiments on the effect of air humidity on the flow properties of glass powders. Powder Technology. 2011, 207, 437-443.

13. Zhang H., Wan Z., Ma D., Zhang B., Zhou P. Coupled effects of moisture content and inherent clay minerals on the cohesive strength of remodeled coal. Energies. 2017, 10, 12 pp.

14. De Bono J.P., McDowell G.R. On the packing and crushing of granular materials. International Journal of Solids and Structures. 2018, 1-8.

15. Castellanos A. The relationship between attractive interparticle forces and bulk behaviour in dry and uncharged fine powders. Advances in Physics. 2005, 54(4), 263-376.

16. Richefeu V., El Youssoufi M.S., Radjai F. Shear strength properties of wet granular materials. Physical Review E. 2006, 73(5), 13 pp.

17. Platonov P., Poltorak V. Investigation of shear of a granular material along a bordering surface. Powder Technology. 1970, 3, 361-363.

18. Bucklin R.A., Molenda M., Bridges T.C., Ross I.J. Slip-stick frictional behavior of wheat on galvanized steel. Transactions of the ASAE. 1996, 39(2), 649-653. 